\title{
Serum LPS and CDI63 Biomarkers Confirming the Role of Gut Dysbiosis in Overweight Patients with NASH
}

This article was published in the following Dove Press journal: Diabetes, Metabolic Syndrome and Obesity: Targets and Therapy

\author{
Mona A Hegazy' \\ Sherif M Mogawer (D) \\ Alshaimaa Rezk L R Alnaggar (D) ${ }^{\prime}$ \\ Olfat A Ghoniem (1D) ${ }^{2}$ \\ Rasha M Abdel Samie' \\ 'Internal Medicine Department, Kasr \\ Alainy Hospital, Faculty of Medicine, \\ Cairo University, Cairo, Egypt; ${ }^{2}$ Internal \\ Medicine, Ministry of Health, Cairo, Egypt
}

Correspondence: Mona A Hegazy Tel+20l00I42I55I

Email monahegazy@cu.edu.eg
Background: Gut-microbiota alterations and bacterial translocation might attribute to hepatic inflammation. Lipopolysaccharide stimulates toll-like receptor 4 leading to the activation of Kupffer cells which express the surface receptor, CD 163.

Objective: To assess the levels of CD 163 and LPS in overweight and obese patients with different degrees of NAFLD as confirmed by liver biopsy (NAS score).

Methods: This is an observational case-control study. Sixty overweight and obese patients with NAFLD and 40 healthy controls were enrolled in the study. Liver biopsy was obtained from all participants with NAFLD. LPS and CD 163 levels were assessed using ELISA.

Results: The mean LPS and CD163 levels were significantly higher in patients with NAFLD when compared with healthy controls ( $p$-value $<0.001$, p-value $<0.001$, respectively). LPS and CD163 levels were the lowest in Non-NASH $(13.17 \pm 3.34,5.61 \pm 2.35 \mathrm{ng} / \mathrm{mL}$, respectively) and the highest in NASH (58.61 $3 \pm 3.81,18.11 \pm 6.84$, respectively) (p-value $<0.001$, p-value $<0.001$, respectively). Statistically significant correlation was found between the levels of LPS and CD163 and NAS score (p-value $<0.001$, p-value $<0.001$, respectively), steatosis grade ( $\mathrm{p}$-value $<0.001, \mathrm{p}$-value $<0.001$, respectively), degree of inflammation ( $\mathrm{p}$ value 0.017 , p-value $<0.001$, respectively) and ballooning ( $r=0.663$, p-value $<0.001, r=$ 0.558 , p-value $<0.001$, respectively). In ROC analysis, both SCD163 and LPS had high sensitivity and specificity in diagnosing NAFLD. CD163 and LPS had the high sensitivity and accuracy in discriminating NASH from Non-NASH (p-value $<0.0001$ in both). Moreover, the mean serum levels of LPS and sCD163 correlated positively and significantly with the BMI ( $r=0.329$, $\mathrm{p}$ value $<0.01 ; \mathrm{r}=0.477$. $\mathrm{p}$ value $<0.001$, respectively).

Conclusion: sCD163 and LPS can be used as non-invasive tools for diagnosis and grading of NAFLD severity in overweight and obese patients, thus confirming the role of dysbiosis in fat deposition and inflammation and suggesting the potential benefits of gut-microbiotatargeted therapies in restoring the gut homeostasis.

Keywords: non-alcoholic steatohepatitis, inflammation, microbiota, CD163, LPS

\section{Introduction}

Non-alcoholic fatty liver disease (NAFLD) is regarded as one of the manifestations of cardiometabolic syndrome comprising diabetes mellitus, dyslipidemia and obesity. ${ }^{1}$ In the USA, NAFLD is currently considered the second most frequent indication for liver transplantation after chronic hepatitis $C{ }^{2}$ NAFLD encompasses a range of pathological changes in the liver which includes simple steatosis, nonalcoholic steatohepatitis, liver cirrhosis and may be hepatocellular carcinoma. ${ }^{2}$ 
Non-alcoholic steatohepatitis (NASH) is distinguished by progressive inflammation that is followed by hepatocyte degenerative changes. ${ }^{2}$

The precise cause of NASH has not been so far clearly elucidated, but studies have proposed the role of the gut microbiota and dysbiosis in the pathogenesis of this disease. ${ }^{3-5}$ Studies on germ-free mice that were fed with high-fat content in diet had lower lipid in the liver compared with conventionally housed mice suggesting a role of germs in NAFLD pathogenesis. ${ }^{6}$ Some bacterial species were found to be associated with NAFLD such as Proteobacteria, Enterobacteria, Escherichia, ${ }^{7}$ and Bacteroides. ${ }^{8}$ Gut microbiota might be implicated in the evolution of NAFLD and NASH and this may be attributable to the translocation of bacteria involved in dysbiosis and their metabolic products to the liver via disrupted gut barrier leading to hepatic inflammation. ${ }^{9,10}$ Obesity is related to alterations in gut bacteria and is also associated with an increase in gut permeability. ${ }^{11}$

Metagenomics studies ${ }^{12-15}$ suggested that gut dysbiosis and its metabolic outcomes modulate the development of obesity in different ways and that it is not only a mere imbalance status of the commensal phyla. ${ }^{16}$ Gut microbiome alteration may change the size and composition of bile acid pool with subsequent altered signaling and activation of bile acid receptors such as farnesoid $\mathrm{X}$ receptor (FXR) and Takeda G protein-coupled receptor 5(TGR5) and hence perturbing lipid and glucose homeostasis. ${ }^{16,17}$ Dysbiosis also alters short-chain fatty acids (SCFA) production with a consequent altered secretion of gut peptide YY(PYY) and glucagon-like peptide1 (GLP-1) thus affecting appetite and satiety. ${ }^{18}$

Lipopolysaccharide (LPS) (Endotoxin) is a microbial molecule found in the basic structure of the Gram-negative bacterial cell wall. ${ }^{19}$ LPS as a pathogen-associated molecular pattern (PAMP) is identified by certain pattern recognition receptor (PRR) called Toll-like Receptor 4. This induces a strong inflammatory response via TLR4 and nuclear factor kappa beta (NF K $\beta$ ) signaling pathway. ${ }^{20}$ Studies on human beings showed elevated LPS levels in obese individuals and also in NAFLD. ${ }^{21}$ These findings suggested that dysbiosis is associated with the development of NAFLD in obese patients. ${ }^{22}$

TLR4 is expressed by various hepatic cells; hence, the effect of LPS is profound. Activation of Kupffer cells via TLR4-dependent mechanisms is associated with the consequent production of TNF-alpha, reactive oxygen species and insulin resistance. ${ }^{23}$
Kupffer cells are hepatic macrophages that express high levels of CD 163. CD 163 is a hemoglobin scavenger receptor that is expressed by the monocytes and macrophages. ${ }^{24}$ It can be found in a soluble form in serum (sCD163) after being shed from the cell surface by certain proteolytic enzymes and is upregulated in conditions with macrophage activation in inflammatory liver diseases. ${ }^{25} \mathrm{CD} 163$ levels are high in hepatitis C, hepatitis $\mathrm{B}$ infection, alcoholic hepatitis and liver cirrhosis with portal hypertension. ${ }^{26}$ However, the relation between CD 163 levels, serum LPS and NAFLD severity has not been studied. ${ }^{27}$ Our hypothesis is that LPS and CD 163 are both associated with NAFLD severity and that they might be used as diagnostic biomarkers that correlate well with liver biopsy findings in overweight and obese NAFLD patients.

The aim of this study is to assess the levels of LPS and CD 163 (representing gut dysbiosis, and hepatic inflammation) patients with NAFLD and to correlate their levels with the presence of NASH in liver biopsy and to emphasize the role of gut dysbiosis in overweight and obese patients.

\section{Patients and Methods}

A total of 60 overweight and obese participants with NAFLD and 40 healthy controls were enrolled during the period from December 2015 to August 2017. Participants were prospectively recruited from Gastroenterology and Hepatology outpatient clinic of Kasr El Ainy Hospital, Cairo University. An informed consent was obtained from all participants before enrolment. The study protocol followed the ethical guidelines of the Declaration of Helsinki $^{28}$ and was approved by Cairo University Hospital Research Ethics Committee (REC) and Institutional review Board (IRB) in 28-5-2011 (N-7-2011).

NAFLD patients included in the study were $\geq 18$ years, overweight or obese with BMI $>25 \mathrm{~kg} / \mathrm{m} 2$ and demonstrated a bright liver on abdominal ultrasound with or without an elevation of liver transaminases. Patients were excluded from the study if they had hepatitis B or C, or other causes of chronic liver disease. Patients with any associated comorbidities as hypertension, diabetes, thyroid disease, malignancy or decompensated liver disease were all excluded from the study. Moreover, patients with a history of alcohol consumption, antibiotic use within the previous 3 months, or drug intake (amiodarone, corticosteroids, tamoxifen, methotrexate, oral contraceptives) were excluded from the study. The control group was identified as being free of any existing or previous liver disease. 
Detailed history taking, complete physical examination including anthropometric measures (weight, height, waist circumference, and BMI was calculated) were obtained from all participants. Subjects were considered overweight if BMI was $25-29.9 \mathrm{~kg} / \mathrm{m}^{2}$ and were defined as obese if BMI was $\geq 30 \mathrm{~kg} / \mathrm{m}^{2}$.

Laboratory investigations included liver and kidney function tests, liver enzymes including transaminases (ALT, AST) and GGT. Fasting plasma glucose, a lipid profile, hepatitis markers (including hepatitis B surface antigen and hepatitis $\mathrm{C}$ virus antibodies), serum LPS and CD 163 were also obtained.

Abdominal ultrasonography was carried out for all participants after an 8 hour-fast in the supine, right and left lateral positions using a Toshiba Apilo XV scanner equipped with a $3.5 \mathrm{MHz}$ curved array probe to detect the presence of liver steatosis. A single operator performed the ultrasound to circumvent inter-observer variability.

The ultrasonographic finding of bright liver confirmed the diagnosis of NAFLD and grading of steatosis was done. ${ }^{22}$

Liver biopsies were obtained, under complete aseptic technique, from all patients with NAFLD. A written consent was obtained. A single pathologist assessed the histological characteristics of the biopsies and they were graded according to the NAFLD activity score (NAS) recommended by the National Institute of Diabetes and Digestive and Kidney Diseases NASH Clinical Research Network and reported as NAFLD activity score (NAS). ${ }^{29}$

NAS score was employed to grade the activity of NAFLD. In patients with NAFLD, the total NAS score denotes the sum of scores for steatosis, lobular inflammation, and ballooning and ranges from 0 to 8 . NAS score of $\geq 5$ strongly matched a diagnosis of "definite NASH", 3 or 4 was considered "borderline NASH", while NAS $\leq 2$ was considered "Non-NASH" 29 The stage of fibrosis was evaluated discretely from NAS using a scale of four points: $0=$ no fibrosis; $1=$ mild/moderate zone 3 perisinusoidal fibrosis or portal/periportal fibrosis only; 2 = perisinusoidal and portal/periportal fibrosis; $3=$ bridging fibrosis and $4=$ cirrhosis. A fibrosis grade of $\geq 2$ was considered as significant fibrosis. ${ }^{30}$

\section{Biochemical Analysis}

Plasma endotoxin (LPS) levels: Samples were diluted with LAL reagent in a ratio of 1:3 and heated at a temperature of $65{ }^{\circ} \mathrm{C}$ for half an hour. All samples were analyzed in replica according to the instructions, using the Limulus
Amoebocyte Lysate (LAL) assay QCL-1000 (Lonza, Valais Switzerland).

Soluble CD163 (sCD163): Blood samples were collected in a 10-mL EDTA-coated tube, centrifuged and serum was then stored at $-80{ }^{\circ} \mathrm{C}$. The samples were thawed and serum sCD163 was analyzed using the Quantikine enzyme-linked immunosorbent assay system (R\&D Systems, Minneapolis, MN). The mean assay coefficient of variance was $3.3 \%$ for sCD163.

\section{Statistical Analysis}

The statistical package SPSS version 21 was used for data coding and entry. Data were analyzed using mean, standard deviation, median, minimum and maximum for quantitative variables and frequencies (number of cases) and relative frequencies (percentages) for categorical variables. The nonparametric Kruskal-Wallis was employed when comparing more than 2 groups of quantitative variables and the Mann-Whitney $U$-test when comparing two groups. Chi-square $(\chi 2)$ test was performed when comparing categorical data, whereas the Exact test was used when the estimated frequency was less than 5 . Odds ratio (OR) with $95 \%$ confidence intervals was calculated. $\mathrm{P}$ value $<0.05$ was considered statistically significant. ${ }^{31,32}$

\section{Results}

Participants were subdivided according to NAS score 33 into three categories: 20 patients with NASH (33.3\%) (18 females and 2 males), 20 patients with borderline NASH (33.3\%) (20 females) and 20 patients who did not have NASH (33.3\%) (19 females and 1 male). Only one patient in the NASH category had fibrosis. The demographic and the laboratory data are demonstrated in Table 1 and Table 2.

The mean levels of LPS level and CD163 were more pronounced in participants with NAFLD when compared with their levels in healthy control subjects (p-value $<0.001$ in both), Figure 1.

When comparing the levels of LPS and CD163 in the three categories of participants with NAFLD, it was found that their mean levels were the lowest in simple steatosis (Non-NASH). The highest levels were found in NASH (pvalue $<0.001$ in both) Figure 2 .

There was a highly significant correlation between the level of LPS and CD163 with BMI ( $r=0.329$, p-value $<0.01, \mathrm{r}=0.477$, $\mathrm{p}$-value $<0.001$, respectively).

Focusing on the liver biopsy findings, LPS and CD163 were significantly associated with steatosis grade $(r=$ 
Table I Demographic and Laboratory Data in Patients and Healthy Controls

\begin{tabular}{|c|c|c|c|}
\hline & NAFLD, Mean \pm SD & Control, Mean \pm SD & $P$ value \\
\hline Age (years) & $43.54 \pm 7.63$ & $43.78 \pm 6.78$ & 0.877 \\
\hline BMI (Kg/m2) & $34.32 \pm 4.46$ & $22.00 \pm 1.56$ & $<0.001$ \\
\hline Waist $(\mathrm{cm})$ & $105.30 \pm 16.48$ & $72.40 \pm 4.31$ & $<0.001$ \\
\hline FBG (mg/dl) & $99.47 \pm 8.94$ & $96.10 \pm 9.88$ & 0.080 \\
\hline $\mathrm{T}-\mathrm{CHOL}(\mathrm{mg} / \mathrm{dl})$ & $204.65 \pm 33.89$ & $156.92 \pm 7.49$ & $<0.001$ \\
\hline LDL (mg/dl) & $106.78 \pm 21.69$ & $86.68 \pm 6.63$ & $<0.001$ \\
\hline $\mathrm{HDL}(\mathrm{mg} / \mathrm{dl})$ & $45.83 \pm 8.97$ & $52.30 \pm 6.98$ & $<0.001$ \\
\hline TG (mg/dl) & $162.62 \pm 46.00$ & $112.88 \pm 15.25$ & $<0.001$ \\
\hline ALT (IU/I) & $30.47 \pm 17.95$ & $16.32 \pm 5.02$ & $<0.001$ \\
\hline AST (IU/I) & $31.75 \pm 19.15$ & $17.55 \pm 4.42$ & $<0.001$ \\
\hline GGT (IU/I) & $41.60 \pm 23.92$ & $31.90 \pm 8.48$ & 0.069 \\
\hline LPS (EU/mL) & $33.37 \pm 28.21$ & $10.78 \pm 3.50$ & $<0.001$ \\
\hline CDI63 (ng/mL) & $12.32 \pm 7.23$ & $3.32 \pm 1.71$ & $<0.001$ \\
\hline
\end{tabular}

Abbreviations: ALT, alanine transaminase; AST, aspartate transaminase; BMI, body mass index; CDI63, cluster of differentiation I63; FBG, fasting blood glucose; GGT, gamma-glutamyl transaminase; HDL, high-density cholesterol; LDL, low-density lipoprotein cholesterol; LPS, lipopolysaccharide; NAFLD, non-alcoholic fatty liver disease; T-CHOL, total cholesterol; TG, triglyceride.

0.634 , p-value $<0.001, \mathrm{r}=0.563$, p-value $<0.001$, respectively), lobular inflammation $(r=0.307$, $\mathrm{p}$-value $<0.001, \mathrm{r}=$ 0.479 , p-value $<0.001$, respectively) and ballooning ( $\mathrm{r}=$ 0.663 , p-value $<0.001, r=0.558$, p-value $<0.001$, respectively). Both markers had statistically significant positive correlation with NAS score $(r=0.765$, p-value $<0.001, r=$ 0.740 , p-value $<0.001$, respectively). (Table 3 ). Moreover, it was found that the levels of CD163 and LPS were correlated with each other $(r=0.613$, p-value $<0.001)$. However, there was no significant correlation between both LPS and CD 163 levels and ALT, AST, GGT and metabolic parameters (Table 3 )

Univariate analysis revealed that LPS and CD163 were significantly correlated with NAFLD (LPS, OR: 1.35, CI: 1.152-1.576, p-value $<0.001$ and CD163, OR: 1.99, CI: 1.455-2.746, p-value $<0.001$ ). (Table 4)

Furthermore, in multiple regression analysis, LPS and CD163 showed a highly significant independent association with borderline NASH and NASH with the high predictive power in discriminating between NASH and Non-NASH (AOR: 1.453, 95\% CI: 1.015-2.078, p-value $=0.04$ for LPS and AOR: 2.16, 95\% CI: $1.283-3.637$ for CD163, p-value $=0.004)$. (Table 4)

We also examined whether CD163 and LPS could be used as reliable biomarkers for the diagnosis of NAFLD. In ROC analysis, CD163 was found to have higher specificity in diagnosing NAFLD with AUC 0.93, 76.7\% sensitivity and $97.5 \%$ specificity as well as high positive predictive power $97.9 \%$, p-value $<0.0001$. However, LPS had higher sensitivity of $86.7 \%$, higher accuracy $89.5 \%$ and negative predictive power $80 \%$, p-value $<0.0001$. Nevertheless, both biomarkers were found to be significant predictors of the disease (Figure 3).

The diagnostic performance of both biomarkers in the different grades of NAFLD according to by NAS score was illustrated using ROC curve in Figure 4. Considering the area under the curve, CD163 was the highest sensitivity and accuracy to discriminate NASH from Non-NASH (AUC 0.981, 95\% CI 98.1-98.1\%, p-value <0.0001). Also, LPS showed higher specificity $100 \%$, but lower sensitivity of $85 \%$ and accuracy of $92.5 \%$, when compared with CD163, for diagnosis of NASH vs Non-NASH (AUC 0.961, 95\% CI 94.3-98\%, p-value <0.0001). Both ROC curves (Figure $4 \mathrm{~B}$ and $\mathrm{E}$ respectively) were closer to the perfect discrimination between NASH and Non-NASH in both biomarkers. Moreover, both LPS and CD163 showed high sensitivity, specificity and accuracy as regards the discrimination between borderline NASH and NonNASH (Figure 4A and D respectively). However, LPS and CD163 did not show good accuracy in discriminating $\mathrm{NASH}$ from borderline NASH (Accuracy: 75\% and $67.5 \%$, respectively) (Figure $4 \mathrm{C}$ and F). (Figure 4).

\section{Discussion}

The exact cause of NASH has not been yet clearly elucidated, but studies have suggested the role of the gut microbiota in the pathogenesis of this disease. ${ }^{3-5}$ LPS is a constituent of the cell wall of Gram-negative bacteria that is continuously released during the death of gutderived bacteria and is a potent inflammatory trigger 
Table 2 Clinical and Laboratory Data in Subgroups of NAFLD Patients (NASH, Borderline NASH and Non-NASH)

\begin{tabular}{|c|c|c|c|c|c|c|}
\hline \multicolumn{3}{|c|}{ According to NAS Score } & $\begin{array}{l}\text { NASH } \\
\text { Mean } \pm \text { SD }\end{array}$ & $\begin{array}{l}\text { Borderline } \\
\text { Mean } \pm \text { SD }\end{array}$ & $\begin{array}{l}\text { Non-NASH } \\
\text { Mean } \pm \text { SD }\end{array}$ & $P$ value \\
\hline \multicolumn{3}{|l|}{ ALT (IU/I) } & $41.10 \pm 23.80$ & $27.65 \pm 11.08$ & $22.65 \pm 10.93$ & 0.008 \\
\hline \multicolumn{3}{|l|}{ AST (IU/I) } & $43.00 \pm 28.43$ & $26.85 \pm 7.68$ & $25.40 \pm 8.40$ & 0.037 \\
\hline \multicolumn{3}{|l|}{ GGT (IU/I) } & $43.50 \pm 21.14$ & $41.10 \pm 18.79$ & $40.20 \pm 31.16$ & 0.465 \\
\hline \multicolumn{3}{|l|}{ NAS } & $5.35 \pm 0.59$ & $3.65 \pm 0.49$ & $2.00 \pm 0.00$ & $<0.001$ \\
\hline \multicolumn{3}{|l|}{ LPS (EU/mL) } & $58.6 I \pm 33.8 I$ & $28.33 \pm 13.80$ & $13.17 \pm 3.34$ & $<0.001$ \\
\hline \multicolumn{3}{|c|}{ CD $163(\mathrm{ng} / \mathrm{mL})$} & $|8.1| \pm 6.84$ & $13.22 \pm 5.15$ & $5.61 \pm 2.35$ & $<0.001$ \\
\hline $\begin{array}{l}\text { Steatosis } \\
\mathrm{G}=\text { grade }\end{array}$ & $\begin{array}{ll}\text { G } & 0 \\
\text { G } & 1 \\
\text { G } & 2 \\
\text { G } & 3\end{array}$ & $\begin{array}{l}I(I .7 \%) \\
3 I(5 I .7 \%) \\
I 2(20.0 \%) \\
I 6(26.7 \%)\end{array}$ & $\begin{array}{l}0 \\
12(60 \%) \\
5(25 \%) \\
3(15 \%)\end{array}$ & $\begin{array}{l}0 \\
0 \\
7(35.0 \%) \\
13(65.0 \%)\end{array}$ & $\begin{array}{l}\mathrm{I}(5.0 \%) \\
19(95.0 \%) \\
0 \\
0\end{array}$ & $<0.001$ \\
\hline Inflammation & $\begin{array}{ll}\text { G } & 0 \\
\text { G } & 1 \\
\text { G } & 2\end{array}$ & $\begin{array}{l}34(56.7 \%) \\
25(4 I .7 \%) \\
I(I .7 \%)\end{array}$ & $\begin{array}{l}\text { I I (55\%) } \\
9(45 \%) \\
0\end{array}$ & $\begin{array}{l}6(30.0 \%) \\
13(65.0 \%) \\
I(5.0 \%)\end{array}$ & $\begin{array}{l}17(85.0 \%) \\
3(15.0 \%) \\
0\end{array}$ & $<0.001$ \\
\hline Ballooning & $\begin{array}{ll}\text { G } & 0 \\
\text { G } & 1 \\
\text { G } & 2\end{array}$ & $\begin{array}{l}3(5 \%) \\
24(40 \%) \\
33(55 \%)\end{array}$ & $\begin{array}{l}0 \\
I(5 \%) \\
19(95 \%)\end{array}$ & $\begin{array}{l}I(5 \%) \\
5(25 \%) \\
\mid 4(70 \%)\end{array}$ & $\begin{array}{l}2(10 \%) \\
18(90 \%) \\
0\end{array}$ & $<0.001$ \\
\hline
\end{tabular}

Abbreviations: ALT, alanine transaminase; AST, aspartate transaminase; CDI63, cluster of differentiation I63; GGT, gamma-glutamyl transaminase; LPS, lipopolysaccharide; NAS, NAFLD activity score; NASH, non-alcoholic steatohepatitis.

signaling through the TLR4/NF- $\kappa \mathrm{B}$ pathway and in turn contributing to metabolic endotoxemia. ${ }^{20}$

CD163 is a haemoglobin scavenger receptor, that is expressed on monocytes and macrophages. ${ }^{34,35}$ In the settings of macrophage activation, its soluble form is shed on the cell surface through cleavage by proteolytic enzymes and then released into the circulation ${ }^{36}$ Kupffer cells, hepatic macrophages, that play a pivotal role in promoting liver inflammation and fibrosis, express very high levels of CD163, hence reflecting the strong association between macrophage activation and chronic hepatic inflammation.37 Moreover, elevated CD163 levels have been linked to the chronic inflammatory state involved in the development of obesity. ${ }^{38,39}$

In the current study, the clinical and metabolic parameters, the liver histology, and the serum levels of LPS and sCD163 in overweight and obese patients with NAFLD and control subjects were assessed. The association of these markers with the presence of NAFLD and its progression from simple steatosis to non-alcoholic steatohepatitis were clarified.

The main findings in this study were the detection of greater levels of serum LPS and sCD163 in overweight and obese NAFLD patients as compared to the healthy controls, as well as its significant correlation with the amount of fat deposited in the liver and the severity of inflammation according to liver biopsy (NAS score).

The present study showed that LPS levels were significantly elevated in overweight and obese participants with NAFLD and were found to correlate significantly with the BMI. This was in accordance with a study by Troseid et al conducted on obese patients undergoing bariatric surgery, that demonstrated significantly higher LPS levels in obese individuals as compared to controls and a markedly significant reduction in serum LPS levels following bariatric surgery. ${ }^{40}$ LPS levels also correlated significantly with cardiometabolic risk factors including the BMI in the latter study.

In addition, many cross-sectional studies ${ }^{41,42}$ have also reported elevated concentrations of circulating endotoxin (LPS) in obese subjects as compared to lean individuals as well as enhanced signaling of TLR-4 in skeletal muscles of obese individuals. ${ }^{43}$ The elevated LPS levels in obese individuals as observed in the present study and in previous studies can be explained by altered gut microbiome (dysbiosis) and an increased gut permeability with a resultant increase in translocation of LPS from the gut into the circulation creating a state of metabolic endotoxemia and 


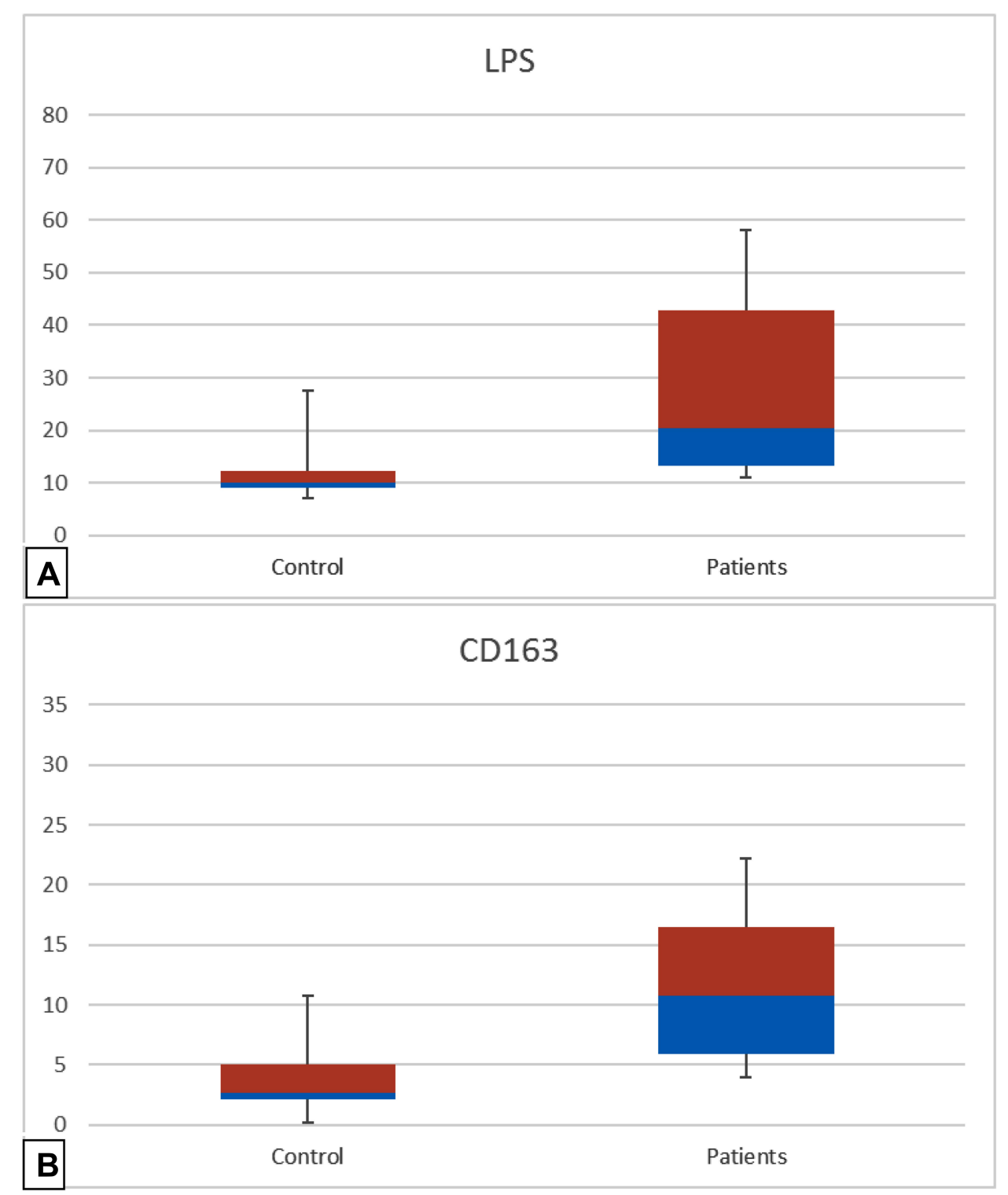

Figure I The serum levels of LPS (sub-figure (A)) and CDI63 (sub-figure (B)) in patients with NAFLD and in control subjects are represented (Median with Interquartile range, $25 \%-75 \%$ ), $\mathrm{p}$-value $<0.00 \mathrm{I}$ in both LPS and CDI63. The data were analyzed by Mann-Whitney Test.

Abbreviations: CDI63, cluster of differentiation 163; LPS, lipopolysaccharides.

hence triggering a low-grade inflammatory state which is observed in obesity. ${ }^{44,45}$

In the current study, a highly significant difference in the serum LPS levels was observed between NAFLD subgroups. The mean serum LPS level was found to be the lowest in patients with Non-NASH and the highest among patients with NASH. A positive correlation between mean LPS levels and the grade of steatosis, degree of inflammation and ballooning on biopsy had also been demonstrated in the present study.

This was in concordance with a previous study by du Plessis et $\mathrm{al}^{46}$ where they found that LPS was increased in patients with NAFLD compared to healthy controls. However, when they compared LPS levels between bariatric patients with limited liver disease (no NAFL and NAFL with NAS score <4) to patients with NASH/ NASH and fibrosis, they found that LPS levels were comparable between the subgroups, and did not also differ in obese subjects with normal histology of the liver as compared to patients with NASH, suggesting that LPS is not the chief factor associated with the progression to $\mathrm{NASH}^{46}$

The present study revealed a highly significant correlation between the mean serum CD163 levels and the BMI. 


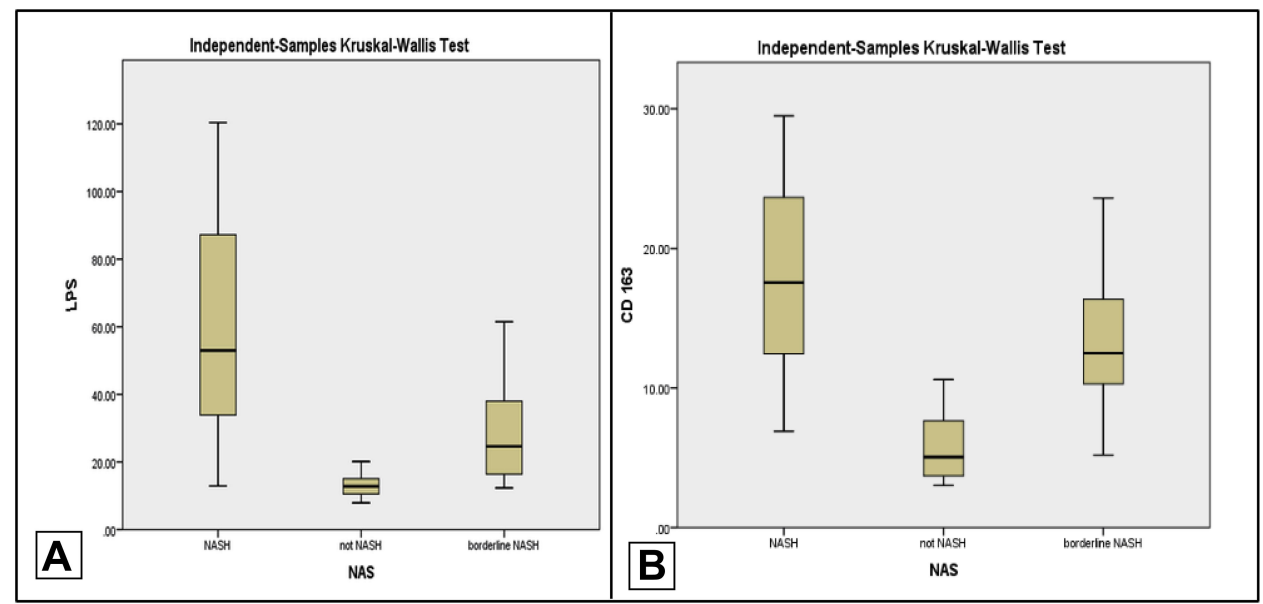

Figure 2 Comparison between the 3 categories of participants with NAFLD, who were classified according to NAS score into NASH, not NASH and borderline NASH as regards the serum levels of LPS in sub-figure (A) $(\mathrm{p}$-value $<0.00 \mathrm{I})$ and $C D I 63$ sub-figure $(\mathbf{B})(\mathrm{p}$-value $<0.00 \mathrm{I})$.

Abbreviations: CD 163, cluster of differentiation 163; LPS, lipopolysaccharides; NAFLD, non-alcoholic fatty liver disease; NAS, NAFLD activity score; NASH, non-alcoholic steatohepatitis.

This is in accordance with various studies that had demonstrated increased sCD163 levels in obese children and adults. ${ }^{47,48}$ In a study by Fjeldborg et al, ${ }^{49}$ serum CD163 levels were elevated in obese healthy subjects, as compared to lean subjects and somewhat normalized after a dietary-induced weight loss by a very low energy diet (VLED) program for 8 weeks. Moreover, CD163 levels showed a positive correlation with BMI and the waist circumference at baseline and were significantly decreased with weight reduction. ${ }^{49}$

The association between CD163 levels and BMI in overweight and obese subjects in the present study was also confirmed in a study by Kazankov et a ${ }^{50}$ conducted on overweight and obese children who were considered for a 10-week period of dietary intervention (healthy diet and an exercise program) and a 12-month follow-up. Serum
ALT levels were elevated in only $47.8 \%$ of the children and $39.3 \%$ had evidence of steatosis as demonstrated by a liver echogenicity score $\geq 1$. CD163 levels were significantly lower after 12 months compared with baseline and at 10 weeks. A strong link was also observed between alterations in SCD163 and improvement in liver enzymes, but there was a weak tendency to an association with BMISD score during lifestyle intervention. ${ }^{30}$

The present study demonstrated a highly significant positive correlation between the mean CD163 levels and the grade of steatosis, degree of inflammation and ballooning. This was in accordance with a study by De Vito et al which revealed a significant association between the intrahepatic expression of $\mathrm{CD} 45+, \mathrm{CD} 3+$ and $\mathrm{CD} 163+$ cells in the portal and lobular regions and all the markers of steatosis, ballooning and fibrosis in a pediatric population with biopsy-proven NAFLD. ${ }^{51}$

Table 3 Correlation of Different Laboratory Parameters and Liver Biopsy and CD 163 and LPS Levels

\begin{tabular}{|l|l|l|}
\hline & Correlation Coefficient for LPS (P-value) & Correlation Coefficient for CD I63 (P-value) \\
\hline CD I63 & $0.613(<0.00 I)$ & 1 \\
BMI $(\mathrm{Kg} / \mathrm{m} 2)$ & $0.329(0.0 \mathrm{I})$ & $0.477(<0.00 \mathrm{I})$ \\
ALT (IU/I) & $0.176(0.179)$ & $0.20 I(0.124)$ \\
AST (IU/I) & $0.204(0.1 \mathrm{I} 8)$ & $0.193(0.14 \mathrm{I})$ \\
GGT (IU/I) & $0.150(0.25 \mathrm{I})$ & $0.179(0.172)$ \\
NAS & $0.765(<0.00 \mathrm{I})$ & $0.740(<0.00 \mathrm{I})$ \\
Steatosis & $0.634(<0.00 \mathrm{I})$ & $0.563(<0.00 \mathrm{I})$ \\
Inflammation & $0.307(0.017)$ & $0.479(<0.00 \mathrm{I})$ \\
Ballooning & $0.663(<0.00 \mathrm{I})$ & $0.558(<0.00 \mathrm{I})$ \\
\hline
\end{tabular}

Abbreviations: ALT, alanine transaminase; AST, aspartate transaminase; BMI, body mass index; CDI63, cluster of differentiation I63; GGT, gamma-glutamyl transaminase; LPS, lipopolysaccharide; NAS, NAFLD activity score. 
Table 4 Univariate Analysis and Multiple Regression Analysis Showing the Predictive Power of Both Biomarkers for Diagnosing NAFLD and Its Grades According to NAS Score

\begin{tabular}{|c|c|c|c|c|}
\hline \multicolumn{2}{|l|}{ Univariate Analysis* } & OR & $95 \% \mathrm{Cl}$ & p-value \\
\hline \multicolumn{2}{|l|}{ LPS } & 1.35 & $1.152-1.576$ & $0.00 I^{* *}$ \\
\hline \multicolumn{2}{|l|}{ CDI63 } & 1.99 & $1.455-2.746$ & $0.00 I^{* *}$ \\
\hline \multicolumn{5}{|c|}{ Multiple Regression Analysis** } \\
\hline & NAS & AOR & $95 \% \mathrm{Cl}$ & $\mathrm{p}$-value \\
\hline \multirow[t]{3}{*}{ LPS } & Not NASH & \multicolumn{3}{|c|}{ I (Reference) } \\
\hline & Borderline NASH & 1.377 & $0.965-1.966$ & 0.07 \\
\hline & NASH & 1.453 & $1.015-2.078$ & 0.04 \\
\hline \multirow[t]{3}{*}{ CDI63 } & Not NASH & \multicolumn{3}{|c|}{ I (Reference) } \\
\hline & Borderline NASH & 1.896 & $1.144-3.142$ & 0.01 \\
\hline & $\mathrm{NASH}$ & 2.160 & $1.283-3.637$ & 0.004 \\
\hline
\end{tabular}

Notes: *Univariate analysis showing the predictive power of LPS (lipopolysaccharide) and CDI63 for NAFLD (Non-alcoholic fatty liver disease). **Multiple regression analysis showing the predictive power of both biomarkers for the diagnosis of NASH and Borderline NASH versus Not-NASH with adjusted OR. $\mathrm{p}$-value calculated depend on logistic regression analysis.

Abbreviations: AOR, adjusted odd ratio; C.I, confidence interval; CDI63, cluster of differentiation I63; LPS, lipopolysaccharide; NASH, non-alcoholic steatohepatitis; NAFLD, non-alcoholic fatty liver disease; OR, odds ratio.

This confirmed the results of a study by Mueller et al which revealed that patients with NAS score $<5$ had significantly higher serum CD163 levels as compared to controls, suggesting a possible link between Kupffer cell activation and inflammation and the timely hepatic lipid accumulation even before being histologically evident. ${ }^{27}$ The latter study had also revealed a significant association between sCD163, NASH and the degree of fibrosis, with the smallest values of sCD163 being observed in healthy obese control subjects (with
$\mathrm{NAS}=0$ ) and the greatest values in those with NASH and advanced fibrosis. SCD163 also correlated with steatosis grade, lobular inflammation, and hepatocyte ballooning. Collectively, this supports the significant role of Kupffer cell activation in the pathogenesis of NASH and fibrosis.

Our study also was in line with other previous studies that reported elevated CD163 in patients with NASH suggesting that activation of the Kupffer cells is a causative agent for hepatic injury in NASH. ${ }^{52-54}$
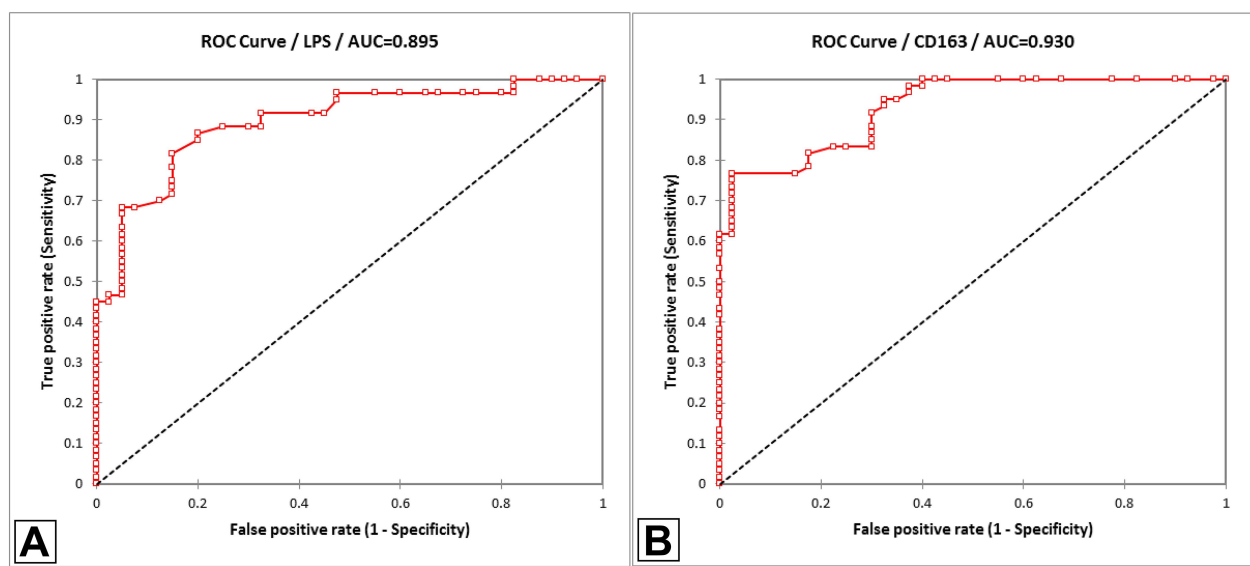

Figure 3 Depicts 2 different ROC curves and shows the diagnostic performances of LPS (sub-figure (A)) and CDI63 (sub-figure (B)) to discriminate patients from control ( $p$-value $<0.000 I)$. Considering the area under the curve (AUC), CDI63 is better than LPS, and the curve is closer to the perfect discrimination. In sub-figure (A): CDI63 was found to have high specificity reaching 97.5\% (AUC: 0.93). In sub-figure (B): LPS was found to have high sensitivity $86.7 \%$ (AUC:0.89).

Abbreviations: AUC, area under the curve; CDI63, cluster of differentiation; LPS, lipopolysaccharides; ROC, receiver operating characteristic curve. 


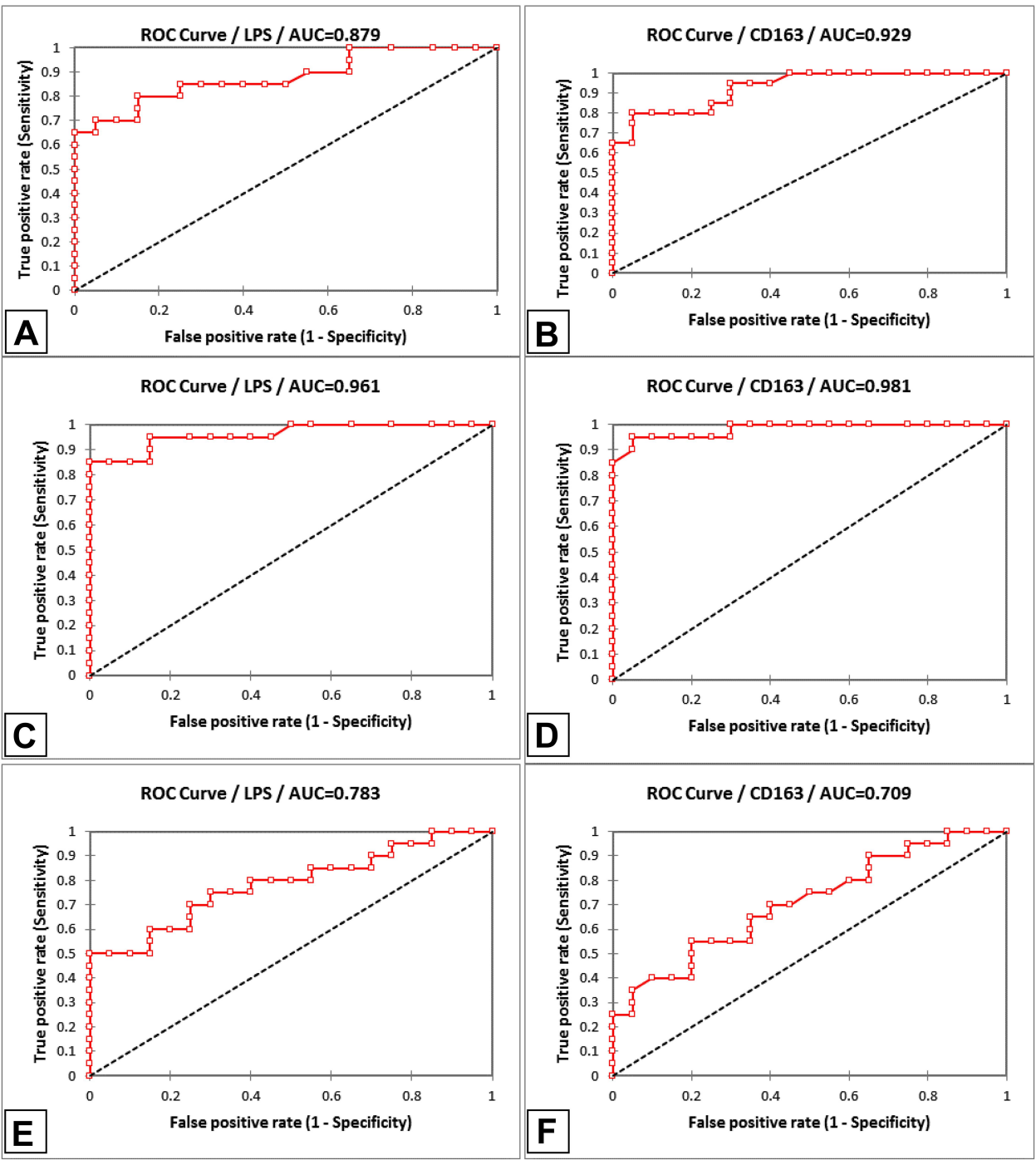

Figure 4 Depicts 6 different ROC curves and shows LPS and CDI63 diagnostic performances to discriminate between NASH, Borderline and Not NASH. In sub-figures (A) (LPS) and (D) (CDI63), LPS and CDI63 showed high sensitivity, specificity and accuracy as regards the discrimination between borderline NASH and Non-NASH, (AUC in LPS: 0.879 , AUC in CDI63: 0.929). CDI63 had the highest sensitivity and accuracy to discriminate NASH from Non-NASH (AUC 0.98I, 95\% Cl 98.I-98.1\%, P-value $<0.000$ I). Also, LPS showed higher specificity $100 \%$, but lower sensitivity of $85 \%$ and accuracy of $92.5 \%$, when compared with CDI63, for diagnosis of NASH vs Non-NASH (AUC 0.96I, 95\% Cl 94.3-98\%, p-value <0.000I). Both ROC curves (sub-figures (B) and (E) respectively) were closer to the perfect discrimination between NASH and Non-NASH in both biomarkers. LPS and CDI63 did not show good accuracy in discriminating NASH from borderline NASH (Accuracy: $75 \%$ and $67.5 \%$ respectively) (subfigure $(\mathbf{C})$ and $(\mathbf{F})$ respectively).

Abbreviations: AUC, area under the curve; LPS, lipopolysaccharides; NASH, non-alcoholic steatohepatitis; ROC, receiver operating curve.

One of the most important results of the present study was the ability of sCD163 and serum LPS levels to discriminate between NASH and Non-NASH. The AUC for serum LPS was 0.96 (95\% CI: 0.94-0.98); the best cut-off point was 20.1 with sensitivity $85 \%$ and specificity $100 \%$. As regards the sCD163, the AUC was 0.98 (95\% CI: 0.97-0.99); the best cut-off point was 10 with sensitivity $95 \%$ and specificity $95 \%$. We can hence conclude that serum LPS and sCD163 can be useful markers in predicting the severity of liver injury.
The association of sCD163 levels with biochemical and histological evidence of NAFLD was further explored in two distinct cohorts of 157 Australian and 174 Italian NAFLD patients. ${ }^{55}$ In non-parametric ROC analysis, SCD163 was $^{2}$ found to be strongly associated with the stage of advanced fibrosis after adjustment for any identifiable risk factors for NAFLD. The study also demonstrated a continuing increase in sCD163 levels corresponding to an increased histologic severity of NAFLD. This association was, however, independent of anthropometric, metabolic and inflammatory measures, proposing a direct link between liver injury and the activation of 
macrophages. This is consistent with the results of the present study which through multivariate analysis also demonstrated the strong predictive power of sCD163 in the diagnosis of borderline NASH and NASH, independent of other parameters, thus confirming the promising capability of sCD163 as a non-invasive marker of disease stage in NAFLD patients. $^{55}$

A strong association between serum LPS and sCD163 levels had been demonstrated in the present study, indicating that there might be a link between macrophage activation by LPS and upregulation of CD163 on their surface and association with NAFLD specifically.

These findings highlighted the clinical implication of manipulating gut microbiota through gut microbiota-targeted therapies in overweight and obese NAFLD, particularly in the setting of accruing benefits of gut-microbiota alteration in clinical and experimental studies by using prebiotics, ${ }^{56-59}$ probiotics, synbiotics, ${ }^{60-63}$ antibiotics and herbal medicines. ${ }^{64,65}$

Several studies showed that the addition of prebiotics like oligofructose (OFS), to the diet of patients with $\mathrm{NASH}$, decreased the levels of LPS and cytokines and lowered the expression of inflammatory and oxidative stress indicators by the liver, in obese and diabetic mice. ${ }^{56}$ As a result, the strategy of gut-microbiota targeted therapies for NAFLD is greatly appreciated in re-establishing gut homeostasis. ${ }^{66}$

The present study emphasized the potential role of serum LPS \& s CD 163 as noninvasive biomarkers for diagnosis and assessment of disease stage in overweight and obese NAFLD patients. Furthermore, they are inexpensive, and highly reproducible markers. This will help in restricting liver biopsy to certain groups of patients and will also assist in early management of NAFLD and follow up of patients to avoid progression to fibrosis and its related complications.

Some of the limitations of our study were the preponderance of the female gender in the groups studied; and only one patient had fibrosis which hindered the study of the association between serum LPS and CD163 and the degree of fibrosis.

In conclusion, CD163 and LPS were significantly correlated with NASH in liver biopsy of overweight and obese NAFLD patients and this confirmed the importance of dysbiosis in hepatic inflammation as well as fat deposition. Moreover, alteration of the gut microbiome could pose a probable therapeutic target for the treatment of obesity. This study strongly recommends that both biomarkers can be used as non-invasive tools for diagnosis and grading of NAFLD severity. Nevertheless, additional studies with a greater population are needed to validate these biomarkers in overweight and obese NAFLD patients and to study the influence of microbiota-targeted therapies on these serological markers and if they can be used to monitor therapeutic response in NAFLD.

\section{Abbreviations}

BMI, body mass index; LPS, lipopolysaccharide; NAS, NAFLD activity score; NASH, non-alcoholic steatohepatitis; NAFLD, non-alcoholic fatty liver disease; PAMP, pathogen-associated molecular pattern; PRR, pattern recognition receptor; TLR4, toll like receptor 4 .

\section{Funding}

No financial support was received.

\section{Disclosure}

The authors report no conflicts of interest for this work.

\section{References}

1. Younossi Z, Anstee QM, Marietti M, et al. Global burden of NAFLD and NASH: trends, predictions, risk factors and prevention. Nat Rev Gastroenterol Hepatol. 2018;15(1):11-20. doi:10.1038/ nrgastro.2017.109

2. Anstee QM, Targher G, Day CP. Progression of NAFLD to diabetes mellitus, cardiovascular disease or cirrhosis. Nat Rev Gastroenterol Hepatol. 2013;10(6):330.

3. Jumpertz R, Le DS, Turnbaugh PJ, et al. Energy-balance studies reveal associations between gut microbes, caloric load, and nutrient absorption in humans. Am J Clin Nutr. 2011;94(1):58-65. doi:10.3945/ajen.110.010132

4. Bäckhed F, Ding H, Wang T, et al. The gut microbiota as an environmental factor that regulates fat storage. Proc Natl Acad Sci. 2004;101 (44):15718-15723. doi:10.1073/pnas.0407076101

5. Csak T, Ganz M, Pespisa J, Kodys K, Dolganiuc A, Szabo G. Fatty acid and endotoxin activate inflammasomes in mouse hepatocytes that release danger signals to stimulate immune cells. Hepatology. 2011;54(1):133-144. doi:10.1002/hep.24341

6. Rabot S, Membrez M, Bruneau A, et al. Germ-free C57BL/6J mice are resistant to high-fat-diet-induced insulin resistance and have altered cholesterol metabolism. FASEB J. 2010;24(12):4948-4959.

7. Zhu L, Baker SS, Gill C, et al. Characterization of gut microbiomes in nonalcoholic steatohepatitis (NASH) patients: a connection between endogenous alcohol and NASH. Hepatology. 2013;57(2):601-609.

8. Boursier J, Mueller O, Barret M, et al. The severity of nonalcoholic fatty liver disease is associated with gut dysbiosis and shift in the metabolic function of the gut microbiota. Hepatology. 2016;63 (3):764-775. doi:10.1002/hep.28356

9. Kolodziejczyk AA, Zheng D, Shibolet O, Elinav E. The role of the microbiome in NAFLD and NASH. EMBO Mol Med. 2019;11(2). doi:10.15252/emmm.201809302

10. Ruiz AG, Casafont F, Crespo J, et al. Lipopolysaccharide-binding protein plasma levels and liver TNF-alpha gene expression in obese patients: evidence for the potential role of endotoxin in the pathogenesis of non-alcoholic steatohepatitis. Obes Surg. 2007;17(10):1374. doi:10.1007/s11695-007-9243-7 
11. De Minicis S, Rychlicki C, Agostinelli L, et al. Dysbiosis contributes to fibrogenesis in the course of chronic liver injury in mice. Hepatology. 2014;59(5):1738-1749. doi:10.1002/hep.26695

12. Armougom F, Henry M, Vialettes B, Raccah D, Raoult D. Monitoring bacterial community of human gut microbiota reveals an increase in Lactobacillus in obese patients and Methanogens in anorexic patients. PLoS One. 2009;4(9):e7125. doi:10.1371/journal.pone. 0007125

13. Turnbaugh PJ, Hamady M, Yatsunenko T, et al. A core gut microbiome in obese and lean twins. Nature. 2009;457(7228):480-484 doi:10.1038/nature07540

14. Walters WA, Xu Z, Knight R. Meta-analyses of human gut microbes associated with obesity and IBD. FEBS Lett. 2014;588(22):42234233. doi:10.1016/j.febslet.2014.09.039

15. Sze MA, Schloss PD. Looking for a signal in the noise: revisiting obesity and the microbiome. MBio. 2016;7(4). doi:10.1128/ mBio.01018-16

16. Tseng $\mathrm{C}-\mathrm{H}, \mathrm{Wu} \mathrm{C}-\mathrm{Y}$. The gut microbiome in obesity. J Formos Med Assoc. 2019;118(Suppl):S3-S9. doi:10.1016/j.jfma.2018.07.009

17. Wang C, Zhu C, Shao L, Ye J, Shen Y, Ren Y. Role of bile acids in dysbiosis and treatment of nonalcoholic fatty liver disease. Mediators Inflamm. 2019;2019:7659509. doi:10.1155/2019/7659509

18. Sun L, Ma L, Ma Y, Zhang F, Zhao C, Nie Y. Insights into the role of gut microbiota in obesity: pathogenesis, mechanisms, and therapeutic perspectives. Protein Cell. 2018;9(5):397-403. doi:10.1007/s13238018-0546-3

19. Soares J-B, Pimentel-Nunes P, Roncon-Albuquerque R, LeiteMoreira A. The role of lipopolysaccharide/toll-like receptor 4 signaling in chronic liver diseases. Hepatol Int. 2010;4(4):659-672. doi:10.1007/s12072-010-9219-x

20. Testro AG, Visvanathan K. Toll-like receptors and their role in gastrointestinal disease. J Gastroenterol Hepatol. 2009;24(6):943954. doi:10.1111/j.1440-1746.2009.05854.x

21. Mouzaki M, Comelli EM, Arendt BM, et al. Intestinal microbiota in patients with nonalcoholic fatty liver disease. Hepatology. 2013;58 (1):120-127. doi:10.1002/hep.26319

22. Plessis J, Korf H, J V P, Windmolders P, Vander I. Pro-inflammatory cytokines but not endotoxin-related parameters associate with disease severity in patients with NAFLD. Plos One. 2016;1-15. DOI:10.1371/journal.pone.0166048

23. Spruss A, Kanuri G, Wagnerberger S, Haub S, Bischoff SC, Bergheim I. Toll-like receptor 4 is involved in the development of fructose-induced hepatic steatosis in mice. Hepatology. 2009;50 (4):1094-1104. doi:10.1002/hep. 23122

24. Møller HJ, Peterslund NA, Graversen JH, Moestrup SK. Identification of the hemoglobin scavenger receptor/CD163 as a natural soluble protein in plasma. Blood. 2002;99(1):378-380. doi:10.1182/blood.V99.1.378

25. Hintz KA, Rassias AJ, Wardwell K, et al. Endotoxin induces rapid metalloproteinase-mediated shedding followed by up-regulation of the monocyte hemoglobin scavenger receptor CD163. J Leukoc Biol. 2002;72(4):711-717.

26. Andersen ES, Rødgaard-Hansen S, Moessner B, Christensen PB, Møller HJ, Weis N. Macrophage-related serum biomarkers soluble CD163 (sCD163) and soluble mannose receptor (sMR) to differentiate mild liver fibrosis from cirrhosis in patients with chronic hepatitis C: a pilot study. Eur J Clin Microbiol Infect Dis. 2014;33(1):117122. doi:10.1007/s10096-013-1936-3

27. Mueller JL, Feeney ER, Bch MB, et al. Circulating soluble CD163 is associated with steatohepatitis and advanced fibrosis in nonalcoholic fatty liver disease. Clini Transl Gastroenterol. 2015;6(10):e114. doi:10.1038/ctg.2015.36

28. Association WM. World Medical Association Declaration of Helsinki: ethical principles for medical research involving human subjects. JAMA. 2013;310(20):2191. doi:10.1001/jama.2013.281053
29. Kleiner DE, Brunt EM, Van Natta M, et al. Design and validation of a histological scoring system for nonalcoholic fatty liver disease. Hepatology. 2005;41(6):1313-1321. doi:10.1002/hep.20701

30. Brunt EM, Kleiner DE, Wilson LA, et al. Portal chronic inflammation in nonalcoholic fatty liver disease (NAFLD): a histologic marker of advanced NAFLD - clinicopathologic correlations from the nonalcoholic steatohepatitis clinical research network. Hepatology. 2009;49 (3):809-820. doi:10.1002/hep.22724

31. Chan YH. Biostatistics 102: quantitative data-parametric \& nonparametric tests. Blood Press. 2003;140(24.08):0-79.

32. Chan YH. Biostatistics 103: qualitative data-tests of independence. Singapore Med J. 2003;44(10):498-503.

33. Brunt EM, Kleiner DE, Wilson LA, Belt P, Neuschwander-Tetri BA. (CRN) NCRN. Nonalcoholic fatty liver disease (NAFLD) activity score and the histopathologic diagnosis in NAFLD: distinct clinicopathologic meanings. Hepatology. 2011;53(3):810-820. doi:10.1002/ hep. 24127

34. Moestrup SK, Møller HJ. CD163: a regulated hemoglobin scavenger receptor with a role in the anti-inflammatory response. Ann Med. 2004;36(5):347-354. doi:10.1080/07853890410033171

35. Møller HJ, de Fost M, Aerts H, Hollak C, Moestrup SK. Plasma level of the macrophage-derived soluble CD163 is increased and positively correlates with severity in Gaucher's disease. Eur J Haematol. 2004;72(2):135-139. doi:10.1046/j.0902-4441.2003.00193.x

36. Weaver LK, Hintz-Goldstein KA, Pioli PA, et al. Pivotal advance: activation of cell surface Toll-like receptors causes shedding of the hemoglobin scavenger receptor CD163. J Leukoc Biol. 2006;80 (1):26-35. doi:10.1189/jlb.1205756

37. Mueller JL, Feeney ER, Zheng H, et al. Circulating soluble CD163 is associated with steatohepatitis and advanced fibrosis in nonalcoholic fatty liver disease. Clin Transl Gastroenterol. 2015;6:e114. doi:10.1038/ctg.2015.36

38. Chawla A, Nguyen KD, Goh YPS. Macrophage-mediated inflammation in metabolic disease. Nat Rev Immunol. 2011;11(11):738-749. doi: $10.1038 /$ nri3071

39. Tam CS, Clément K, Baur LA, Tordjman J. Obesity and low-grade inflammation: a paediatric perspective. Obes Rev. 2010;11(2):118126. doi:10.1111/j.1467-789X.2009.00674.x

40. Trøseid M, Nestvold TK, Rudi K, Thoresen H, Nielsen EW, Lappegård KT. Plasma lipopolysaccharide is closely associated with glycemic control and abdominal obesity: evidence from bariatric surgery. Diabetes Care. 2013;36(11):3627-3632. doi:10.2337/dc130451

41. Kallio KAE, Hätönen KA, Lehto M, Salomaa V, Männistö S, Pussinen PJ. Endotoxemia, nutrition, and cardiometabolic disorders. Acta Diabetol. 2015;52(2):395-404. doi:10.1007/s00592-0140662-3

42. Basu S, Haghiac M, Surace P, et al. Pregravid obesity associates with increased maternal endotoxemia and metabolic inflammation. Obesity (Silver Spring). 2011;19(3):476-482. doi:10.1038/oby.2010.215

43. Frisard MI, McMillan RP, Marchand J, et al. Toll-like receptor 4 modulates skeletal muscle substrate metabolism. Am J Physiol Endocrinol Metab. 2010;298(5):E988-98. doi:10.1152/ ajpendo.00307.2009

44. Delzenne NM, Neyrinck AM, Cani PD. Modulation of the gut microbiota by nutrients with prebiotic properties: consequences for host health in the context of obesity and metabolic syndrome. Microb Cell Fact. 2011;10(Suppl 1):S10. doi:10.1186/1475-2859-10-S1-S10

45. Cani PD, Amar J, Iglesias MA, et al. Metabolic endotoxemia initiates obesity and insulin resistance. Diabetes. 2007;56(7):1761-1772. doi:10.2337/db06-1491

46. Du Plessis J, Korf H, van Pelt J, et al. Pro-inflammatory cytokines but not endotoxin-related parameters associate with disease severity in patients with NAFLD. PLoS One. 2016;11(12):e0166048. doi:10.1371/journal.pone.0166048 
47. Santen RJ, Kagan R, Altomare CJ, Komm B, Mirkin S, Taylor HS. Current and evolving approaches to individualizing estrogen receptor-based therapy for menopausal women. J Clin Endocrinol Metab. 2014;99(3):733-747. doi:10.1210/jc.2013-3680

48. Parkner T, Sørensen LP, Nielsen AR, et al. Soluble CD163: a biomarker linking macrophages and insulin resistance. Diabetologia. 2012;55(6):1856-1862. doi:10.1007/s00125-012-2533-1

49. Fjeldborg K, Christiansen T, Bennetzen MJ, Møller H, Pedersen SB, Richelsen B. The macrophage-specific serum marker, soluble CD163, is increased in obesity and reduced after dietary-induced weight loss. Obesity. 2013;21(12):2437-2443. doi:10.1002/oby.20376

50. Kazankov K, Møller HJ, Lange A, et al. The macrophage activation marker sCD163 is associated with changes in NAFLD and metabolic profile during lifestyle intervention in obese children. Pediatr Obes. 2015;10(3):226-233. doi:10.1111/ijpo.252

51. De Vito R, Alisi A, Masotti A, et al. Markers of activated inflammatory cells correlate with severity of liver damage in children with nonalcoholic fatty liver disease. Int J Mol Med. 2012;30(1):49-56. doi:10.3892/ijmm.2012.965

52. Baffy G. Kupffer cells in non-alcoholic fatty liver disease: the emerging view. J Hepatol. 2009;51(1):212-223. doi:10.1016/j.jhep.2009.03.008

53. Park J-W, Jeong G, Kim SJ, Kim MK, Park SM. Predictors reflecting the pathological severity of non-alcoholic fatty liver disease: comprehensive study of clinical and immunohistochemical findings in younger Asian patients. J Gastroenterol Hepatol. 2007;22(4):491497. doi:10.1111/j.1440-1746.2006.04758.x

54. Fotiadu A, Gagalis A, Akriviadis E, et al. Clinicopathological correlations in a series of adult patients with non-alcoholic fatty liver disease. Pathol Int. 2010;60(2):87-92. doi:10.1111/j.1440-1827.2009.02489.x

55. Kazankov K, Barrera F, Møller HJ, et al. The macrophage activation marker sCD163 is associated with morphological disease stages in patients with non-alcoholic fatty liver disease. Liver Int. 2016;36 (10):1549-1557. doi:10.1111/liv.13150

56. Cani PD, Possemiers S, Van de Wiele $\mathrm{T}$, et al. Changes in gut microbiota control inflammation in obese mice through a mechanism involving GLP-2-driven improvement of gut permeability. Gut. 2009;58(8):1091-1103.

57. Matsumoto K, Ichimura M, Tsuneyama K, et al. Fructo-oligosaccharides and intestinal barrier function in a methionine-choline-deficient mouse model of nonalcoholic steatohepatitis. PLoS One. 2017;12(6): e0175406. doi:10.1371/journal.pone.0175406
58. Pachikian BD, Essaghir A, Demoulin J, et al. Prebiotic approach alleviates hepatic steatosis: implication of fatty acid oxidative and cholesterol synthesis pathways. Mol Nutr Food Res. 2013;57(2):347359. doi:10.1002/mnfr.201200364

59. Dewulf EM, Cani PD, Claus SP, et al. Insight into the prebiotic concept: lessons from an exploratory, double blind intervention study with inulin-type fructans in obese women. Gut. 2013;62 (8):1112-1121. doi:10.1136/gutjnl-2012-303304

60. Eslamparast T, Poustchi H, Zamani F, Sharafkhah M, Malekzadeh R, Hekmatdoost A. Synbiotic supplementation in nonalcoholic fatty liver disease: a randomized, double-blind, placebo-controlled pilot study. Am J Clin Nutr. 2014;99(3):535-542.

61. Malaguarnera M, Vacante M, Antic T, et al. Bifidobacterium longum with fructo-oligosaccharides in patients with non alcoholic steatohepatitis. Dig Dis Sci. 2012;57(2):545-553. doi:10.1007/s10620-0111887-4

62. Mofidi F, Poustchi H, Yari Z, et al. Synbiotic supplementation in lean patients with non-alcoholic fatty liver disease: a pilot, randomised, double-blind, placebo-controlled, clinical trial. Br J Nutr. 2017;117 (5):662-668. doi:10.1017/S0007114517000204

63. Asgharian A, Askari G, Esmailzade A, Feizi A, Mohammadi V. The effect of symbiotic supplementation on liver enzymes, C-reactive protein and ultrasound findings in patients with non-alcoholic fatty liver disease: a clinical trial. Int J Prev Med. 2016;7.

64. Zhong L-J, Xie Z-S, Yang H, Li P, Xu X-J. Moutan Cortex and Paeoniae Radix Rubra reverse high-fat-diet-induced metabolic disorder and restore gut microbiota homeostasis. Chin J Nat Med. 2017;15 (3):210-219. doi:10.1016/S1875-5364(17)30037-7

65. Xu J, Chen H-B, Li S-L. Understanding the molecular mechanisms of the interplay between herbal medicines and gut microbiota. Med Res Rev. 2017;37(5):1140-1185. doi:10.1002/med.21431

66. Ma J, Zhou Q, Li H. Gut microbiota and nonalcoholic fatty liver disease: insights on mechanisms and therapy. Nutrients. 2017;9 (10):1124. doi:10.3390/nu9101124

\section{Publish your work in this journal}

Diabetes, Metabolic Syndrome and Obesity: Targets and Therapy is an international, peer-reviewed open-access journal committed to the rapid publication of the latest laboratory and clinical findings in the fields of diabetes, metabolic syndrome and obesity research. Original research, review, case reports, hypothesis formation, expert opinion and commentaries are all considered for publication. The manuscript management system is completely online and includes a very quick and fair peer-review system, which is all easy to use. Visit http://www.dovepress.com/testimonials.php to read real quotes from published authors. 